Table 1.

astrocytoma
(subependymal astrocytoma
$\begin{aligned} & \mid \\ & \text { ependymoma } \\ & \text { (subependymal oligodendroglioma) } \\ & \text { oligodendroglioma }\end{aligned}$

Table 2.

\begin{tabular}{ll}
\hline malignant astrocytoma & astroblastoma \\
& malignant astrocytoma \\
& anaplastic astrocytoma \\
& glioblastoma multiforme \\
& spongioblastoma polare \\
& astrocytoma grade II-IV \\
malignant oligodendroglioma & oligodendroblastoma \\
malignant ependymoma & ependymoblastoma \\
& ependymoma grade II-IV \\
glioblastoma & glioblastoma multiforme \\
\hline
\end{tabular}

of the benign form. Therefore "malignant astrocytoma" includes not only astroblastoma, anaplastic astrocytoma, some of polar spongioblastoma but also most of so-called glioblastoma. In "malignant ependymoma" all ependymomas with malignant features such as polymorphism, mitotic figure, necrosis are included, and so-called neuroepithelioma are also included in this group.

\title{
Discussion to d-9.
}

\section{Evidence of Cilia and Concentric Lamellation of Glial Processes in Human Astrocytoma}

\author{
Eiichi Tani, Toshio Ametani, Yasuo Kawamura and Hajime Handa \\ Department of Neurosurgery Kyoto University Medical School
}

Simplification of glioma classification is much better. For example, most of astrocytomas, fibrillary or protoplasmic, have fibrillary, protoplasmic, and gemistocytic astrocytes. In addition, there are transitional or intermediate forms of three classes of astrocytes in most tumors.

Specimens shown here were taken from an astrocytoma in the subcortical area of the temporal lobe on the right side. The presence of cilia has been eported in the cells of ependymoma and colloid cyst. Single cilia are evident in 
the perinuclear cytoplasm of protoplasmic or gemistocytic astrocytes. Isolated cilia originate deep in the cell, close to nucleus, and project away into a narrow channel of extracellular space formed by a deep invagination of the cell membrane. The surface membrane or sheath of each cilium is a direct continuation of the cell membrane of the cell from which it arises. In cross sections, eight peripheral fibers and one central fiber may be counted. Some of the central fiber are found in an eccentric position. Basal body and its adnexae are seen at the basal end of the cilium. $n$ associated centriole is located deeper in the cytoplasm than the basal body proper, and suggests that single cilia in astrocytoma have a twocentriole basal organization. The ciliated tumor cells are distinctively not ependymoma but astrocytoma cells from standpoint of light and electron microscopies.

Parallel or concentric lamellation of cellular processes of astrocytoma is visible. In case of concentric lamellation, the centers of these figures are cytoplasmic processes of tumor cells or extracellular space. Many of lamellated figures are formed internally of complete, circular lamellae which are partially enclosed externally by one or more processes disposed about the circumference. When several figures are closely associatd, an attenuated cellular process occasionally surrounds completly two or three mall laminated structures. If the attenuatin of cytoplasmic processes become remarkable, two cell membranes appear to be fused to form a dense line. The extracellular space between the lamellated processes is clear or shows in places a flocculent or fibrillar structures which seem to be similar in appearance to those in the extracellular space. The parallel or concentric lamellation of cellular processes was reported to be characteristic of oligodendroglioma and to suggest that oligodendroglia is responsible for the formation of myelin sheath. However, it seems interesting to see the similar lamellation of cytoplasmic processes in astrocytoma.

\title{
d-10. Precancerous Stages of Experimental Brain Tumors
}

\author{
Hajime Handa, Eiichi Tani, Yasuo Kawamura, Toshio Ametani, \\ Sumihiro Yamashita and Jyuji Takeuchi \\ Department of Neurosurgery, Kyoto University Medical School
}

Masahiro Azuma

Laboratory of Electron microscopy, Tenri Hospital

Ikuta reported a characteristic appearance of virus-like particles in cytoplasmas of reactive cells during the precancerous stages of the lesions induced by 3-methylcholanthrene (MC), 1,2,5,6-dibenzanthracene and benzpyrene.

We implanted 20-methylcholanthrene powder in the right parietal regions of $\mathrm{C} 3 \mathrm{H}$ strain, maintained in the animal colony of Montefiore Hospital and Kyoto University and examined sequence of morphological changes of small lesions around the carcinogen with the aid of electron microscopy. 